\title{
$\rho$-Exponential Stabilization for Caplygin Systems: Simplified Controllability Condition and Examples
}

\author{
Ti-Chung Lee \\ Department of Electrical Engineering \\ Ming Hsin University of Science and Technology \\ Hsinchu, Taiwan 304, R.O.C. \\ tc1120@ms19.hinet.net
}

\author{
Jing-Sin Liu \\ Institute of Information Science \\ Academia Sinica \\ Taipei, Taiwan 115, R.O.C. \\ liu@iis.sinica.edu.tw
}

\begin{abstract}
The paper investigates the global $\rho$ exponential stabilizability for nonholonomic Caplygin systems that are linear in certain state variables. A simple and easily verified controllability condition is proposed to guarantee the global $\rho$-exponential stabilizability. For an important subclass of Caplygin systems, the controllability condition is further reduced to some conditions relating to the degree and non-zero property of the lowest nonzero polynomials in the Taylor expansion of constraint function. A new feature in this paper is that all parameters can be explicitly determined from the constraint function. Moreover, a novel coordinate transformation between Caplygin systems can be used to enhance the proposed criterion so that it can be applied to various situations. Several interesting examples, including of the rolling wheel system and a set-point problem of hopping robot, are provided to validate the effectiveness of the proposed results.
\end{abstract}

Index Terms-Caplygin systems. Coordinate transformation. Decomposition. $\rho$-exponential stabilizability. Controllability condition.

\section{INTRODUCTION}

The paper investigates the global $\rho$-exponential stabilizability of Caplygin systems that can be described as follows:

$$
\begin{aligned}
\ddot{q}_{1} & =u \\
\dot{q}_{2} & =-J\left(q_{1}\right) \dot{q}_{1},
\end{aligned}
$$

where $q_{1} \in \mathfrak{R}^{n}, q_{2} \in \mathfrak{R}^{m}, u \in \mathfrak{R}^{n}$ and the constrain function $J$ is a matrix-valued analytic function defined on $\mathfrak{R}^{n}[2]$. The target of this paper is to propose a simple and easily verified criterion.

Caplygin systems as a subclass of nonholonomic systems were introduced in [2] for the control community. Practical examples includes the knife-edge, the extended power form, the rolling wheel and the hopping robot systems, e.t.c., [2], [3], [6], [9], [13]. In recent years, the interests for such systems follow from the fact that they cannot be stabilized by static time-invariant continuous controllers [4]. Simultaneously, there are no time-varying smooth controllers that can exponentially stabilize them. To overcome these obstacles, several approaches, such as the homogeneous and the discontinuous feedback methods, were proposed [1], [6], [7], [12], [14], [15]. See the survey paper [8] and numerous references therein.

Among these results, the homogeneous feedback method usually needs a special construction for the design of controllers and thus can only be applied to certain specific systems. By contrast, the discontinuous feedback approach can be used in a large class of nonholonomic systems [1], [14], [15]. However, its weakness is that the proposed controllers become very large when the initial state is near a singular hyperplane. In [10], this drawback was further improved by employing non-zero exponentially decaying divisors in the $\sigma$-processing. While the derived controllers are smooth and can guarantee some kind of exponential stability, the equilibrium point is loss of Lyapunove stability.

Quite recently, another kind of continuous controllers was proposed to guarantee the global $\rho$-exponential stabilizability for a class of cascaded systems including of Caplygin systems in the form (1)-(2) [11]. The proposed criterion provides a systematic way to verify the $\rho$ exponential stabilizability. But, due to the criterion depending on the choices of certain parameters, it is not so trivial to determine that a given nonholonomic system is or is not $\rho$-exponentially stabilizable based on the approaches given in that paper. Thus, it deserves a further improvement. This paper toward this direction and would like to propose a simple and direct criterion to verify $\rho$-exponential stabilizability of Caplygin systems. Indeed, a decomposition of the partial state $q_{1}$ and the input $u$ will be given first. The constraint function $J$ will be assumed to be linear in partial state variables of $q_{1}$. Then, a simplified controllability condition can be proposed to guarantee the global $\rho$-exponential stabilizability based on the result of [11]. A new feature in this paper is that all parameters can be explicitly determined from the constraint function $J$ when compares with the previous paper. Moreover, a novel coordinate transformation between Caplygin systems can be used to enhance the proposed criterion so that it can be applied to various situations. For an important subclass of Caplygin systems, the controllability condition will be further reduced to certain easily tested conditions relating to the degree and non-zero property of constraint function $J$. Several interesting examples, including of the rolling wheel systems and a set-point control problem for an extended system of hopping robot, will be provided to validate the effectiveness of the proposed results. From these applications, it can be seen that the proposed criterion does provide a direct and easily checked condition with respect to the results given in present literature in determining whether a Caplygin system can be exponentially stabilized. 


\section{BRIEF REVIEW OF NEWLY DEVELOPED CRITERION}

In this section, a criterion given in [11] will be reviewed briefly. It can be viewed as a preliminary result in our study of the exponential stabilizability for Caplygin systems.

Consider the following cascaded system

$$
\begin{aligned}
& \dot{x}_{1}=A_{1} x_{1}+B_{1} u_{1} \\
& \dot{x}_{2}=A_{2}\left(x_{1}, u_{1}\right) x_{2}+B_{2}\left(x_{1}, u_{1}\right) u_{2},
\end{aligned}
$$

where $x_{i} \in \mathfrak{R}^{n_{i}}$ and $u_{i} \in \mathfrak{R}^{m_{i}}, \forall i=1,2 ; A_{1}$ and $B_{1}$ are matrices with suitable dimensions; $A_{2}\left(x_{1}, u_{1}\right)$ and $B_{2}\left(x_{1}, u_{1}\right)$ are matrix-valued analytic functions [11]. Throughout this paper, let $\mathfrak{R}^{\hat{n} \times \hat{m}}$ denote the set of all $\hat{n} \times \hat{m}$ matrices and $D_{r}$ denote the diagonal matrix with diagonal elements taken from the elements of a vector $r$ in order.

Let us recall and extend some notations from [11]. Let $A: \mathfrak{R}^{\bar{n}} \rightarrow \mathfrak{R}^{\hat{n} \times \hat{m}}$ be a matrix-valued analytic function.

Definition 1. For any $1 \leq i \leq \hat{n}$ and $1 \leq j \leq \hat{m}$, let $p_{i j}^{A}$ denote the lowest nonzero homogeneous polynomial in the Taylor expansion of the $(i, j)$ entry of $A$ at the origin and $d_{i j}^{A}$ denote the degree of $p_{i j}^{A}$. When $p_{i j}^{A} \equiv 0$ (i.e., the $(i, j)$ entry of $A$ is the zero function), let $d_{i j}^{A}=\infty$. Moreover, denote $\tilde{d}_{i}^{A}=\min _{1 \leq j \leq \hat{m}} d_{i j}^{A}$ and $d^{A}=\left(\tilde{d}_{1}^{A}, \tilde{d}_{2}^{A}, \cdots, \tilde{d}_{\hat{n}}^{A}\right)^{T}$.

Definition 2. Let $(r, s) \in \mathfrak{R}^{\hat{n}} \times \mathfrak{R}^{\hat{m}}$ be any integer-valued vector satisfying $r_{i} \leq s_{j}+d_{i j}^{A}, \forall i, \forall j$. Let $\bar{p}_{i j}^{r s A}$ be defined as follows:

$$
\bar{p}_{i j}^{r s A}= \begin{cases}p_{i j}^{A}, & \text { if } r_{i}=s_{j}+d_{i j}^{A}, \\ 0, & \text { if } r_{i}<s_{j}+d_{i j}^{A} .\end{cases}
$$

The definitions of dilation operation, homogeneous norm and global $\rho$-exponential stability are recalled as follows [11]-[12].

Definition 3. Let $v=\left(v_{1}, v_{2}, \cdots, v_{\hat{n}}\right)^{T} \in \mathfrak{R}^{\hat{n}}$. A dilation $\Delta_{\zeta}^{r}: \mathfrak{R}^{\hat{n}} \rightarrow \mathfrak{R}^{\hat{n}}$ on $\mathfrak{R}^{\hat{n}}$ is defined by assigning $\hat{n}$ real numbers $r=\left(r_{1}, r_{2}, \cdots, r_{\hat{n}}\right)^{T}$ and a nonzero real number $\zeta$ such that $\Delta_{\zeta}^{r} v=\left(\zeta^{r_{1}} v_{1}, \zeta^{r_{2}} v_{2}, \cdots, \zeta^{r_{\hat{n}}} v_{\hat{n}}\right)$. Similarly, let $A=\left(a_{i j}\right) \in \mathfrak{R}^{\hat{n} \times \hat{m}}$. A dilation $\Delta_{\zeta}^{r s}: \mathfrak{R}^{\hat{n} \times \hat{m}} \rightarrow \mathfrak{R}^{\hat{n} \times \hat{m}}$ on $\mathfrak{R}^{\hat{n} \times \hat{m}}$ is defined by assigning $\hat{n}+\hat{m}$ real numbers $r=\left(r_{1}, r_{2}, \cdots, r_{\hat{n}}\right)^{T}$ and $s=\left(s_{1}, s_{2}, \cdots, s_{\hat{m}}\right)^{T}$, and a nonzero real number $\zeta$ such that $\Delta_{\zeta}^{r s} A=\left(\zeta^{r_{i}-s_{j}} a_{i j}\right)$

Definition 4. A positive definite continuous function $\rho: \mathfrak{R}^{\hat{n}} \rightarrow \mathfrak{R}$ is called a homogeneous norm w. r. t. the dilation $\Delta_{\zeta}^{r}$ if $\rho\left(\Delta_{\zeta}^{r} x\right)=\zeta \rho(x), \forall \zeta \neq 0, \forall x \in \mathfrak{R}^{\hat{n}}$.

Definition 5. The equilibrium point $x=0$ is globally $\rho$ exponentially stable if there exist a homogeneous norm $\rho$ and two positive constants $\sigma_{1}$ and $\sigma_{2}$ such that for any solution $x$, the following inequality holds:

$$
\rho(x(t)) \leq \sigma_{1} \rho\left(x\left(t_{0}\right)\right) e^{-\sigma_{2}\left(t-t_{0}\right)}, \quad \forall t \geq t_{0} .
$$

To state the main result of [11], we need the following hypotheses.

(H1) There exists an integer-valued vector $(r, s) \in \mathfrak{R}^{n_{2}} \times \mathfrak{R}^{m_{2}}$ satisfying the following inequalities

$$
r_{i} \leq r_{j}+d_{i j}^{A_{2}} \text { and } r_{i} \leq s_{\widetilde{j}}+d_{i \tilde{j}}^{B_{2}}, \forall i, \forall j, \forall \tilde{j}
$$

(H2) For some positive constant $k$ and some vector $(a, b) \in \mathfrak{R}^{n_{1}} \times \mathfrak{R}^{m_{1}}$ satisfying $\left(k I+A_{1}\right) a+B_{1} b=0$, the pair $\left(A_{1}, B_{1}\right)$ and $\left(\bar{A}_{2}(a, b), \bar{B}_{2}(a, b)\right)$ are both controllable where $\bar{A}_{2}=k D_{r}+\left(\bar{p}_{i j}^{r A_{2}}\right)$ and $\bar{B}_{2}=\left(\bar{p}_{i \tilde{j}}^{r s B_{2}}\right)$.

The following result was proven in [11].

Proposition 1. Consider a system of the form (3)-(4). Suppose that (H1)-(H2) hold for some positive constant $k$ and some integer-valued vector $(r, s) \in \mathfrak{R}^{n_{2}} \times \mathfrak{R}^{m_{2}}$. Let $\hat{r}=(\underbrace{1, \ldots, 1}_{n_{1}}, r^{T})^{T} \in \mathfrak{R}^{n_{1}+n_{2}}$ and $K_{1} \in \mathfrak{R}^{m_{1} \times n_{1}}$ and $K_{2} \in \mathfrak{R}^{m_{2} \times n_{2}}$ be two matrices such that the matrices $k I+A_{1}+B_{1} K_{1}$ and $\bar{A}_{2}(a, b)+\bar{B}_{2}(a, b) K_{2}$ are both stable. Then, the origin of the closed-loop system is globally $\rho$-exponentially stable when the controller $\left(u_{1}, u_{2}\right)$ is chosen as follows

$$
\begin{aligned}
& u_{1}=\left(b-K_{1} a\right) \lambda+K_{1} x_{1}, \\
& u_{2}=\left\{\begin{array}{l}
\Delta_{\lambda}^{s r} K_{2} x_{2}, \text { if }\left(x_{1}\left(t_{0}\right), x_{2}\left(t_{0}\right)\right) \neq 0 \\
0, \quad \text { if }\left(x_{1}\left(t_{0}\right), x_{2}\left(t_{0}\right)\right)=0,
\end{array}\right.
\end{aligned}
$$

for any $t_{0} \geq 0$ where $\lambda=e^{-k\left(t-t_{0}\right)} \rho\left(x_{1}\left(t_{0}\right), x_{2}\left(t_{0}\right)\right)$ with $\rho$ being any homogeneous norm w.r.t. dilation $\Delta_{\zeta}^{\hat{r}}$.

\section{CONTROLlability CONDITION AND $\rho$-EXPONENTIAL}

\section{STABILIZABILITY}

\section{A. A Simplified Controllability Condition}

In this subsection, Caplygin systems will be transformed into the form of (3)-(4) under a mild assumption. Simultaneously, it will be shown that $(\mathrm{H} 1)$ holds by a suitable choice of dilation vector $(r, s)$. To verify (H2), a simplified controllability condition will be proposed. Then, the global $\rho$-exponential stabilizability can be guaranteed based on Proposition 1.

First, consider the following assumption.

(C1) (Linear in partial state variables). Suppose the state vector $q_{1}$ and the input vector $u$ can be decomposed as $q_{1}=\left[z_{1}{ }^{T} z_{2}{ }^{T}\right]^{T}$ and $u=\left[\begin{array}{ll}u_{1}^{T} & u_{2}^{T}\end{array}\right]^{T}$ so that the constraint function $J$ can be described as $J\left(z_{1}, z_{2}\right)=\left[\sum_{j=1}^{\bar{n}_{2}} J_{1 j}\left(z_{1}\right) z_{2 j} \quad \bar{J}_{2}\left(z_{1}\right)\right] \quad$ where $z_{1} \in \mathfrak{R}^{\bar{n}_{1}}$, $z_{2}=\left(z_{21}, z_{22}, \cdots, z_{2 \bar{n}_{2}}\right)^{T} \in \mathfrak{R}^{\bar{n}_{2}} \quad, \quad u_{1} \in \mathfrak{R}^{\bar{n}_{1}}, u_{2} \in \mathfrak{R}^{\bar{n}_{2}} \quad$, $\bar{n}_{1}+\bar{n}_{2}=n \quad$, and $J_{1 j}: \mathfrak{R}^{\bar{n}_{1}} \rightarrow \mathfrak{R}^{m \times \bar{n}_{1}}, \quad \forall 1 \leq j \leq \bar{n}_{2} \quad$, and $\bar{J}_{2}: \mathfrak{R}^{\bar{n}_{1}} \rightarrow \mathfrak{R}^{m \times \bar{n}_{2}}$ being matrix-valued analytic functions. 
For the compactness, we define a matrix-valued function $\bar{J}_{1}: \mathfrak{R}^{\bar{n}_{1}} \times \mathfrak{R}^{\bar{n}_{1}} \rightarrow \mathfrak{R}^{m \times \bar{n}_{2}}$ as follows

$$
\bar{J}_{1}\left(z_{1}, z_{3}\right)=\left[\begin{array}{llll}
J_{11}\left(z_{1}\right) z_{3} & J_{12}\left(z_{1}\right) z_{3} & \cdots & J_{1 \bar{n}_{2}}\left(z_{1}\right) z_{3}
\end{array}\right],
$$

$\forall z_{1} \in \Re^{\bar{n}_{1}}, \forall z_{3} \in \mathfrak{R}^{\bar{n}_{1}}$. By the definition, $\bar{J}_{1}$ is linear in $z_{3}$ and satisfies the following equation:

$$
J\left(q_{1}\right) \dot{q}_{1}=\bar{J}_{1}\left(z_{1}, \dot{z}_{1}\right) z_{2}+\bar{J}_{2}\left(z_{1}\right) \dot{z}_{2} .
$$

By the definitions of $J_{1 j}, \bar{J}_{1}$ and $\bar{J}_{2}$, it can be seen that

$$
\tilde{d}_{i}^{J}=\min _{1 \leq j \leq \bar{n}_{2}}\left(\tilde{d}_{i}^{J_{1 j}}+1, \tilde{d}_{i}^{\bar{J}_{2}}\right), \tilde{d}_{i}^{\bar{J}_{1}}=\min _{1 \leq j \leq \bar{n}_{2}} \tilde{d}_{i}^{J_{1 j}}+1, \forall 1 \leq i \leq m .
$$

Let $\bar{d}_{i}=\min \left(\tilde{d}_{i}^{\bar{J}_{1}}, \tilde{d}_{i}^{\bar{J}_{2}}\right), \forall 1 \leq i \leq m$. Then, we have

$$
d^{J}=\left(\bar{d}_{1}, \bar{d}_{2}, \cdots, \bar{d}_{m}\right)^{T}=: \bar{d}
$$

where $d^{J}$ is the "degree vector" of $J$ defined as in Definition 1.

Let $x_{1}=\left[\begin{array}{ll}z_{1}^{T} & \dot{z}_{1}^{T}\end{array}\right]^{T} \in \mathfrak{R}^{n_{1}}$ and $x_{2}=\left[\begin{array}{lll}q_{2}^{T} & z_{2}^{T} & \dot{z}_{2}^{T}\end{array}\right]^{T} \in \mathfrak{R}^{n_{2}}$ with $n_{1}=2 \bar{n}_{1}$ and $n_{2}=m+2 \bar{n}_{2}$. Then, (1)-(2) can be rewritten into the form of (3)-(4) where the matrices $A_{1}$ and $B_{1}$, and the matrix-valued functions $A_{2}$ and $B_{2}$ can be described as follows:

$$
A_{1}=\left[\begin{array}{ll}
0 & I \\
0 & 0
\end{array}\right], B_{1}=\left[\begin{array}{l}
0 \\
I
\end{array}\right], A_{2}=\left[\begin{array}{ccc}
0 & -\bar{J}_{1} & -\bar{J}_{2} \\
0 & 0 & I \\
0 & 0 & 0
\end{array}\right] \text { and } B_{2}=\left[\begin{array}{l}
0 \\
0 \\
I
\end{array}\right] \text {. }
$$

It is easy to see that $\left(A_{1}, B_{1}\right)$ is in the controllable canonical form (CCF) [5]. By the direct computation, it can be checked that the "degree matrices" of $A_{2}$ and $B_{2}$ can be described as follows

$$
\left(d_{i j}^{A_{2}}\right)=\left[\begin{array}{ccc}
\infty & \left(d_{i j_{1}}^{\bar{J}_{1}}\right) & \left(d_{i j_{2}}^{\bar{J}_{2}}\right) \\
\infty & \infty & 0 \\
\infty & \infty & \infty
\end{array}\right] \quad \text { and }\left(d_{\tilde{i} j}^{B_{2}}\right)=\left[\begin{array}{l}
\infty \\
\infty \\
0
\end{array}\right]
$$

Choose the dilation vector $(r, s)$ as follows

$$
r=(\bar{d}_{1}+1, \bar{d}_{2}+1, \cdots, \bar{d}_{m}+1, \underbrace{1, \cdots, 1}_{2 \bar{n}_{2}})^{T}, s=(\underbrace{1,1, \cdots, 1}_{\bar{n}_{2}})^{T} .
$$

Let $E_{\bar{d}} \in \mathfrak{R}^{m \times \bar{n}_{2}}$ be a matrix defined as $E_{\bar{d}}=[\underbrace{\bar{d} \bar{d} \cdots \bar{d}}_{\bar{n}_{2}}]$.

Then, the following inequalities hold:

$$
\left(r_{i}-r_{j}\right)=\left[\begin{array}{lrr}
* & \mathrm{E}_{\bar{d}} & \mathrm{E}_{\bar{d}} \\
-\mathrm{E}_{\bar{d}}^{T} & 0 & 0 \\
-\mathrm{E}_{\bar{d}}^{T} & 0 & 0
\end{array}\right] \leq\left(d_{i j}^{A_{2}}\right), \quad\left(r_{i}-s_{\tilde{j}}\right)=\left[\begin{array}{l}
E_{\bar{d}} \\
0 \\
0
\end{array}\right] \leq\left(d_{i j}^{B_{2}}\right),
$$

by the definition of $\bar{d}$. Hypothesis (H1) follows from the inequalities above.

In the following, let us compute the matrix-valued functions $\bar{A}_{2}$ and $\bar{B}_{2}$. To this end, define two matrixvalued functions as follows:

$$
\bar{P}_{1}\left(z_{1}\right)=\left(\bar{p}_{i j_{1}}^{\bar{d} 0 \bar{J}_{1}}\left(z_{1}, z_{1}\right)\right) \text { and } \bar{P}_{2}\left(z_{1}\right)=\left(\bar{p}_{i j_{2}}^{\bar{d} 0 \bar{J}_{2}}\left(z_{1}\right)\right)
$$

for all $z_{1}$ in $\Re^{\bar{n}_{1}}$ where $\bar{p}_{i \hat{j}}^{\bar{d} 0 \bar{J}_{\hat{j}}}, \hat{j}=1,2$, is the function defined in (5) with $r=\bar{d}$ and $s=0$. Notice that for any positive constant $k$, every solutions $(a, b) \in \mathfrak{R}^{2 \bar{n}_{1}} \times \mathfrak{R}^{\bar{n}_{1}}$ satisfying the equation $\left(k I+A_{1}\right) a+B_{1} b=0$ can be described as $a=\left[\eta^{T},-k \eta^{T}\right]^{T}$ and $b=k^{2} \eta$ for all $\eta \in \mathfrak{R}^{\bar{n}_{1}}$ in view of the form of $A_{1}$ and $B_{1}$ given in (13). By (9), it can be seen that

$$
\left(\bar{p}_{i j_{1}}^{\bar{d} 0 \bar{J}_{1}}(\eta,-k \eta)\right)=-k\left(\bar{p}_{i j_{1}}^{\bar{d} 0 \bar{J}_{1}}(\eta, \eta)\right)=-k \bar{P}_{1}(\eta), \forall \eta \in \mathfrak{R}^{\bar{n}_{1}} .
$$

Notice that the functions $\bar{p}_{i j}^{r r A_{2}}$ and $\bar{p}_{i \tilde{j}}^{r s B_{2}}$ are nonzero functions and equal to $p_{i j}^{A_{2}}$ and $p_{i \tilde{j}}^{B_{2}}$, respectively, only if inequalities (16) became the equalities. Thus, it can be directly checked that

$$
\bar{B}_{2}=\left(\bar{p}_{i \tilde{j}}^{r s B_{2}}\right)=\left[\begin{array}{lll}
0 & 0 & I
\end{array}\right]^{T} .
$$

In view of (17)-(18), we have

$$
\begin{aligned}
& \left(\bar{p}_{i j}^{r r A_{2}}(\eta,-k \eta)\right)= \\
& {\left[\begin{array}{ccc}
0 & -\left(p_{i j_{1}}^{\bar{d} 0 \bar{J}_{1}}(\eta,-k \eta)\right) & -\left(p_{i j_{2}}^{\bar{d} 0 \bar{J}_{2}}(\eta)\right) \\
0 & 0 & I \\
0 & 0 & 0
\end{array}\right]=\left[\begin{array}{ccc}
0 & k \bar{P}_{1}(\eta) & -\bar{P}_{2}(\eta) \\
0 & 0 & I \\
0 & 0 & 0
\end{array}\right]}
\end{aligned}
$$

for all $k>0$ and all $\eta \in \mathfrak{R}^{\bar{n}_{1}}$. Thus,

$$
\bar{A}_{2}=k D_{r}+\left(\bar{p}_{i j}^{r r A_{2}}(\eta,-k \eta)\right)=k I+\left[\begin{array}{ccc}
k D_{\bar{d}} & k \bar{P}_{1}(\eta) & -\bar{P}_{2}(\eta) \\
0 & 0 & I \\
0 & 0 & 0
\end{array}\right] \text {. }
$$

To check the controllability of the pair $\left(\bar{A}_{2}, \bar{B}_{2}\right)$, the following condition is necessary.

(C2) (Reduced order controllability). Suppose $\left(D_{\bar{d}}, \bar{P}\left(\eta_{0}\right)\right)$ is a controllable pair for some $\eta_{0} \in \mathfrak{R}^{\bar{n}_{1}}$ where

$$
\bar{P}=\bar{P}_{1}-D_{\bar{d}} \bar{P}_{2} \text {. }
$$

The following lemma is useful. Its proof is not difficult and omitted here.

Lemma 1. Consider three matrices $A \in \mathfrak{R}^{m \times \bar{n}}, B \in \mathfrak{R}^{m \times p}$ and $C \in \mathfrak{R}^{\bar{n} \times \bar{n}}$. Suppose the matrix $C$ is invertible and $\operatorname{rank}(B)=m$. Then, the following equality holds:

$$
\operatorname{rank}\left[\begin{array}{cc}
A & B \\
C & 0
\end{array}\right]=m+\bar{n} .
$$

Under condition (C2), the controllability of $\left(\bar{A}_{2}, \bar{B}_{2}\right)$ can be guaranteed as follows.

Lemma 2. Suppose (C2) holds. Then, $\left(\bar{A}_{2}\left(\eta_{0}\right), \bar{B}_{2}\right)$ is controllable where $\bar{B}_{2}=\left[\begin{array}{lll}0 & 0 & I\end{array}\right]^{T}$ and $\bar{A}_{2}$ is the matrixvalued function defined in (21).

Proof. Since the elementary column operations do not affect the rank of a matrix, the following equality relating to the controllability matrix can be derived $\operatorname{rank}\left[\bar{B}_{2}, \bar{A}_{2}^{0} \bar{B}_{2},\left(\bar{A}_{2}^{0}\right)^{2} \bar{B}_{2}, \cdots,\left(\bar{A}_{2}^{0}\right)^{m+1} \bar{B}_{2}\right]=\operatorname{rank}\left[\bar{B}_{2}, \bar{B}_{3}, \bar{B}_{4}, \cdots, \bar{B}_{m+3}\right]$, where $\bar{A}_{2}^{0}=\bar{A}_{2}\left(\eta_{0}\right)$,

$$
\begin{aligned}
& \bar{B}_{3}=\bar{A}_{2}^{0} \bar{B}_{2}-k \bar{B}_{2}=\left[\begin{array}{l}
-\bar{P}_{2}\left(\eta_{0}\right) \\
I \\
0
\end{array}\right], \bar{B}_{4}=(1 / k)\left(\bar{A}_{2}^{0} \bar{B}_{3}-k \bar{B}_{3}\right)=\left[\begin{array}{l}
\bar{P}\left(\eta_{0}\right) \\
0 \\
0
\end{array}\right] \text { and } \\
& \bar{B}_{i+4}=(1 / k)\left(\bar{A}_{2}^{0} \bar{B}_{i+3}-k \bar{B}_{i+3}\right)=\left[\begin{array}{l}
D_{\bar{d}}^{i} \bar{P}\left(\eta_{0}\right) \\
0 \\
0
\end{array}\right], \quad \forall 1 \leq i \leq m-1 .
\end{aligned}
$$


Since $\left[\begin{array}{ll}0 & I \\ I & 0\end{array}\right]$ is invertible and

$\left[\bar{B}_{2}, \bar{B}_{3}, \bar{B}_{4}, \cdots, \bar{B}_{m+3}\right]=\left[\begin{array}{cccccc}0 & -\bar{P}_{2}\left(\eta_{0}\right) & \bar{P}\left(\eta_{0}\right) & D_{\bar{d}} \bar{P}\left(\eta_{0}\right) & \cdots & D_{\bar{d}}^{m-1} \bar{P}\left(\eta_{0}\right) \\ 0 & I & 0 & 0 & \cdots & 0 \\ I & 0 & 0 & 0 & \cdots & 0\end{array}\right]$,

the controllability of $\left(\bar{A}_{2}\left(\eta_{0}\right), \bar{B}_{2}\right)$ follows from $(\mathrm{C} 2)$ and Lemma 1. This completes the proof of the lemma.

The following theorem can be proposed based on the previous discussions.

Theorem 1. Consider a Caplygin system of the form (1)-(2). Suppose (C1)-(C2) hold for some $\eta_{0} \in \mathfrak{R}^{\bar{n}_{1}}$. Let $\bar{d}$ be the vector defined in (12). For any positive constant $k$, let $K_{1}=\left[K_{11} K_{12}\right] \in \mathfrak{R}^{\bar{n}_{1} \times \bar{n}_{1}} \times \mathfrak{R}^{\bar{n}_{1} \times \bar{n}_{1}}$ and $K_{2}=\left[K_{21} K_{22} K_{23}\right] \in \mathfrak{R}^{\bar{n}_{2} \times m} \times \Re^{\bar{n}_{2} \times n_{2}} \times \Re^{\bar{n}_{2} \times \bar{n}_{2}}$ be two matrices such that

$\left[\begin{array}{cc}k I & I \\ K_{11} & k I+K_{12}\end{array}\right]$ and $k I+\left[\begin{array}{ccc}k D_{\bar{d}} & k \bar{P}_{1}\left(\eta_{0}\right) & -\bar{P}_{2}\left(\eta_{0}\right) \\ 0 & 0 & I \\ K_{21} & K_{22} & K_{23}\end{array}\right]$

are both stable. Choose the controller $\left(u_{1}, u_{2}\right)$ as follows

$u_{1}=\left(k^{2} I-K_{11}+k K_{12}\right) \eta_{0} \lambda+K_{11} z_{1}+K_{12} \dot{z}_{1}$

and

$u_{2}=\left\{\begin{array}{lc}K_{21}\left(\Delta_{1 / \lambda}^{\bar{d}} q_{2}\right)+K_{22} z_{2}+K_{23} \dot{z}_{2}, \text { if }\left(q_{1}\left(t_{0}\right), \dot{q}_{1}\left(t_{0}\right), q_{2}\left(t_{0}\right)\right) \neq 0, \\ 0, & \text { if }\left(q_{1}\left(t_{0}\right), \dot{q}_{1}\left(t_{0}\right), q_{2}\left(t_{0}\right)\right)=0 .\end{array}\right.$

where $\lambda=e^{-k\left(t-t_{0}\right)} \rho\left(q_{1}\left(t_{0}\right), \dot{q}_{1}\left(t_{0}\right), q_{2}\left(t_{0}\right)\right)$ with $\rho$ being any homogeneous norm w.r.t. dilation vector $\tilde{r}=(\underbrace{1,1, \cdots, 1}_{2 n}, \bar{d}_{1}+1, \bar{d}_{2}+1, \cdots, \bar{d}_{m}+1)^{T}$. Then, the origin of the closed-loop system is globally $\rho$-exponentially stable.

Proof. Based on previous discussions and Lemma 2, hypotheses (H1)-(H2) hold for $a=\left[\eta_{0}{ }^{T}-k \eta_{0}{ }^{T}\right]^{T}, b=k^{2} \eta_{0}$ and the dilation vector $(r, s)$ defined as in (15). Moreover, any homogeneous norm $\rho$ w.r.t. $\Delta_{\tilde{\xi}}^{\tilde{r}}$ can be written as a homogeneous norm $\hat{\rho}$ w.r.t. $\Delta_{\zeta}^{\hat{r}}$ where $\hat{r}=(\underbrace{1, \ldots, 1}_{2 \bar{n}_{1}}, r)$ and $\hat{\rho}\left(x_{1}, x_{2}\right)=\rho\left(\left[\begin{array}{ll}z_{1}^{T} & z_{2}^{T}\end{array}\right]^{T},\left[\begin{array}{ll}\dot{z}_{1}^{T} & \dot{z}_{2}^{T}\end{array}\right]^{T}, q_{2}\right)$ for all $x_{1}=\left[\begin{array}{ll}z_{1}^{T} & \dot{z}_{1}^{T}\end{array}\right]^{T}$ and all $x_{2}=\left[\begin{array}{lll}q_{2}^{T} & z_{2}^{T} & \dot{z}_{2}^{T}\end{array}\right]^{T}$. Notice that the matrices given in (23) are equal to $k I+A_{1}+B_{1} K_{1}$ and $\bar{A}_{2}+\bar{B}_{2} K_{2}$, respectively. Thus, it remains to show that the controllers given in (8) can be written into the form (24)-(25) based on Proposition 1. Using the fact that $a=\left[\eta_{0}^{T},-k \eta_{0}^{T}\right]^{T}, \quad b=k^{2} \eta_{0}$, $x_{1}=\left[\begin{array}{ll}z_{1}^{T} & \dot{z}_{1}^{T}\end{array}\right]^{T}$ and $K_{1}=\left[\begin{array}{ll}K_{11} & K_{12}\end{array}\right]$, it can be directly computed that

$u_{1}=\left(b-K_{1} a\right) \lambda+K_{1} x_{1}=\left(k^{2} I-K_{11}+k K_{12}\right) \eta_{0} \lambda+K_{11} z_{1}+K_{12} \dot{z}_{1}$.

Thus, the first controller $u_{1}$ given in (8) can be written into the form of (24). Since $x_{2}=\left[\begin{array}{llll}q_{2}^{T} & z_{2}^{T} & \dot{z}_{2}^{T}\end{array}\right]^{T}$ and $K_{2}=\left[K_{21} K_{22} K_{23}\right]$, the following equations hold:
$\Delta_{\lambda}^{s r} K_{2} x_{2}=\left[\begin{array}{lll}\Delta_{\lambda}^{0 \bar{d}} K_{21} & K_{22} & K_{23}\end{array}\right]\left[\begin{array}{c}q_{2} \\ z_{2} \\ \dot{z}_{2}\end{array}\right]=\Delta_{\lambda}^{0 \bar{d}} K_{21} q_{2}+K_{22} z_{2}+K_{23} \dot{z}_{2}$,

according to the definitions of $(r, s)$ in $(15)$. Let $K_{21}=\left(k_{i j}\right)$. Then,

$\Delta_{\lambda}^{0 \bar{d}} K_{21}=\left(\lambda^{-\bar{d}_{j}} k_{i j}\right)=\left[\begin{array}{cccc}\lambda^{-\bar{d}_{1}} k_{11} & \lambda^{-\bar{d}_{2}} k_{12} \cdots & \lambda^{-\bar{d}_{m}} k_{1 m} \\ \lambda^{-\bar{d}_{1}} k_{21} & \lambda^{-\bar{d}_{2}} k_{22} \cdots & \lambda^{-\bar{d}_{m}} k_{2 m} \\ \vdots & \cdots & \vdots \\ \lambda^{-\bar{d}_{1}} k_{\bar{n}_{2}} & \lambda^{-\bar{d}_{2}} k_{\bar{n}_{2}} \cdots & \lambda^{-\bar{d}_{m}} k_{\bar{n}_{2} m}\end{array}\right]$.

In view of the equation above, it can be seen that $\Delta_{\lambda}^{0 \bar{d}} K_{21} q_{2}=K_{21} \Delta_{1 / \lambda}^{\bar{d}} q_{2}$. Hence, we have $\Delta_{\lambda}^{s r} K_{2} x_{2}=K_{21} \Delta_{1 / \lambda}^{\bar{d}} q_{2}+K_{22} z_{2}+K_{23} z_{3}$. Particularly, $u_{2}$ can be written into the form of (25) and the theorem follows from Proposition 1.

\section{B. Degree Criterion and Examples}

In this subsection, Theorem 1 will be used to study an important subclass of Caplygin systems. A further simplified criterion related the degree of constrained function will be proposed. Two examples will be given and discussed.

In the following, let us assume that (C1) holds with $\bar{n}_{2}=1$. Under this assumption, a simple criterion can be proposed to check (C2) as follows.

Proposition 2. Consider a Caplygin system of the form (1)(2). Suppose (C1) holds with $\bar{n}_{2}=1$. Let $\bar{d}$ be the vector defined in (12) and $\bar{P}(\eta)=\left(a_{1}(\eta), a_{2}(\eta), \cdots, a_{m}(\eta)\right)^{T}, \forall \eta \in \mathfrak{R}^{\bar{n}_{1}}$, be the function defined in (22). Then, condition (C2) is true if and only if the following conditions hold.
(a) There exists a vector $\eta_{0} \in \mathfrak{R}^{\bar{n}_{1}}$ so that $a_{i}\left(\eta_{0}\right) \neq 0, \forall 1 \leq i \leq m$.
(b) $\bar{d}_{i} \neq \bar{d}_{j}, \forall i \neq j$.

In addition that $J=\left[0 \bar{J}_{2}\right]$ (i.e., $\left.J_{11} \equiv 0\right)$, (C2) is equivalent to the following conditions.
(c) There exists a vector $\eta_{0} \in \mathfrak{R}^{\bar{n}_{1}} \quad$ so that $p_{i 1}^{\bar{J}_{2}}\left(\eta_{0}\right) \neq 0, \forall 1 \leq i \leq m$.

(d) $\tilde{d}_{i}^{\bar{J}_{2}}, \forall 1 \leq i \leq m$, are all distinct positive integers.

Proof. The determinant of the controllability matrix of $\left(D_{\bar{d}}, \bar{P}\left(\eta_{0}\right)\right)$ can be explicitly computed as follows:

$$
\begin{aligned}
& \operatorname{det}\left(\left[\bar{P}, D_{\bar{d}} \bar{P}, \cdots, D_{\bar{d}}^{m-1} \bar{P}\right]\right)=\operatorname{det}\left[\begin{array}{cccc}
a_{1} & \bar{d}_{1} a_{1} & \cdots & \bar{d}_{1}^{m-1} a_{1} \\
a_{2} & \bar{d}_{2} a_{2} & \cdots & \bar{d}_{2}^{m-1} a_{2} \\
\vdots & \vdots & \ddots & \vdots \\
a_{m} & \bar{d}_{m} a_{m} & \cdots & \bar{d}_{m}^{m-1} a_{m}
\end{array}\right] \\
& =\left(\prod_{i=1}^{m} a_{i}\right) \operatorname{det}\left[\begin{array}{cccc}
1 & \bar{d}_{1} & \cdots & \bar{d}_{1}^{m-1} \\
1 & \bar{d}_{2} & \cdots & \bar{d}_{2}^{m-1} \\
\vdots & \vdots & \ddots & \vdots \\
1 & \bar{d}_{m} & \cdots & \bar{d}_{m}^{m-1}
\end{array}\right]=\left(\prod_{i=1}^{m} a_{i}\right) \prod_{1 \leq i<j \leq m}\left(\bar{d}_{i}-\bar{d}_{j}\right) .
\end{aligned}
$$


by using the property of Vandermode matrix [5]. This implies that (C2) holds if and only if conditions (a) and (b) hold. In case of $J=\left[\begin{array}{ll}0 & \bar{J}_{2}\end{array}\right]$, we have $\bar{J}_{1}=0, \bar{P}_{1}=0$ and $\bar{d}_{i}=\tilde{d}_{i}^{\bar{J}_{2}}, \forall 1 \leq i \leq m$, by the definition of $\bar{d}_{i}$. This implies

$$
\bar{P}_{2}=\left(\bar{p}_{i 1}^{\bar{d} 0 \bar{J}_{2}}\right)=\left(p_{i 1}^{\bar{J}_{2}}\right)
$$

and

$$
\bar{P}=-D_{\bar{d}} \bar{P}_{2}=\left(-\bar{d}_{1} p_{11}^{\bar{J}_{2}},-\bar{d}_{2} p_{21}^{\bar{J}_{2}}, \cdots,-\bar{d}_{m} p_{m 1}^{\bar{J}_{2}}\right)^{T} .
$$

Then, (a) is equivalent to (c) and $\bar{d}_{i} \neq 0, \forall 1 \leq i \leq m$. This particularly shows that (a)-(b) is equivalent to (c)-(d) when $J=\left[\begin{array}{ll}0 & \bar{J}_{2}\end{array}\right]$. It completes the proof of the proposition.

In the following, let us consider the set-point problem of a hopping robot as an application.

Example 1. (Hopping robot). Consider a hopping robot system as follows (see Fig. 1) [13]:

$$
\begin{aligned}
& \dot{\psi}=\omega, \\
& \dot{l}=v, \\
& \dot{\theta}=-\frac{m_{l}(l+1)^{2}}{1+m_{l}(l+1)^{2}} \omega,
\end{aligned}
$$

where $(\psi, l, \theta)$ denote the body angle, leg extension, and leg angle of the robot; $m_{l}$ is the mass of the leg at the foot; $\omega$ and $v$ are the velocities of $\psi$ and $l$, respectively. Let $\tau$ and $T$ denote the torque and the force, respectively. Then, we have

$$
\dot{\omega}=\tau / J, \dot{v}=F / M,
$$

where $J$ and $M$ represent the inertial mass and the mass, respectively.

The so-called set-point problem is to find a controller $(\tau, F)$ so that every trajectory $(\psi, l, \theta, \omega, v)$ of system (26)(27) converges to a specific target $(\psi, l, \theta, \omega, v)=\left(\psi_{0}, l_{0}, \theta_{0}, 0,0\right)$ for some non-negative constant $l_{0}$. Define the error and control variables as follows:

$$
\begin{aligned}
& z_{1}=l-l_{0}, z_{2}=\psi-\psi_{0}, q_{1}=\left[z_{1}, z_{2}\right]^{T}, \\
& q_{2}=\theta-\theta_{0}+\left(\psi-\psi_{0}\right) m_{l}\left(l_{0}+1\right)^{2} /\left[1+m_{l}\left(l_{0}+1\right)^{2}\right],
\end{aligned}
$$

and $u_{1}=F / M, u_{2}=\tau / J, u=\left[u_{1}, u_{2}\right]^{T}$.

Since $\lim _{t \rightarrow \infty}\left(q_{1}(t), q_{2}(t), \dot{q}_{1}(t)\right)=0 \quad$ is equivalent to $\lim _{t \leftarrow \infty}(\psi(t), l(t), \theta(t), \omega(t), v(t))=\left(\psi_{0}, l_{0}, \theta_{0}, 0,0\right)$, the set-point problem is reduced to a stability problem. Moreover, the error system can be transformed into a Caplygin system of the form (1)-(2) with $m=1$. The constraint function can be described as $J=\left[\begin{array}{ll}0 & \bar{J}_{2}\left(z_{1}\right)\end{array}\right]$, with

$$
\bar{J}_{2}=\frac{m_{l}\left(z_{1}+l_{0}+1\right)^{2}}{1+m_{l}\left(z_{1}+l_{0}+1\right)^{2}}-\frac{m_{l}\left(l_{0}+1\right)^{2}}{1+m_{l}\left(l_{0}+1\right)^{2}} .
$$

Then, (C1) holds with $\bar{n}_{1}=\bar{n}_{2}=1$ and $J_{11}=0$. Notice that $p_{11}^{\bar{J}_{2}}=2 z_{1} m_{l}\left(l_{0}+1\right) /\left[1+m_{l}\left(l_{0}+1\right)^{2}\right]^{2}$ and thus $\tilde{d}_{1}^{\bar{J}_{2}}=1$. Then, conditions (c)-(d) hold for any $\eta_{0} \neq 0$ and the origin of the error system is globally $\rho$-exponentially stabilizable according to Theorem 1 and Proposition 2. That is to say that the set-point control problem can be solved via the controller (24)-(25) by employing Theorem 1.
In the following, let us discuss the restriction of $(\mathrm{C} 2)$ by a practical example.

Example 2. (Rolling wheel). Consider a rolling wheel system as follows [3]:

$$
\begin{aligned}
& \dot{y}_{1}=y_{5}, \dot{y}_{2}=y_{6}, \dot{y}_{3}=-y_{5} \cos \left(y_{2}\right), \dot{y}_{4}=y_{5} \sin \left(y_{2}\right), \\
& \dot{y}_{5}=v_{1} / 2, \dot{y}_{6}=v_{2},
\end{aligned}
$$

where $y_{i}$ is state variable, $\forall 1 \leq i \leq 6$, and $v_{j}$ is control variable, $\forall 1 \leq j \leq 2$. Consider the following coordinate transformation:

$$
\begin{aligned}
& z_{1}=y_{2}, z_{2}=y_{1}, q_{1}=\left[z_{1}, z_{2}\right]^{T}, q_{2}=\left[y_{3}, y_{4}\right]^{T}, \\
& u_{1}=v_{2}, u_{2}=v_{1} / 2, u=\left[u_{1}, u_{2}\right]^{T} .
\end{aligned}
$$

Then, (30) can be transformed into a Caplygin system of the form (1)-(2) with $m=2$ and the constraint function

$$
J=\left[\begin{array}{cc}
0 & \cos \left(z_{1}\right) \\
0 & -\sin \left(z_{1}\right)
\end{array}\right] .
$$

Thus, (C1) holds with $\bar{n}_{1}=\bar{n}_{2}=1, \quad J_{11} \equiv 0 \quad$ and $\bar{J}_{2}=\left[\cos \left(z_{1}\right),-\sin \left(z_{1}\right)\right]^{T}$. To verify (C2), it is necessary to check conditions (c)-(d) in Proposition 2. However, condition (d) in Proposition 2 does not hold since $p_{11}^{\bar{J}_{2}}=1$ and $\tilde{d}_{1}^{\bar{J}_{2}}=0$. This indicates the restriction of $(\mathrm{C} 2)$. In next subsection, an interesting coordinate transformation will be proposed to enhance $(\mathrm{C} 2)$ so that Theorem 1 can be applied to this system.

\section{Second Form of Caplygin Systems}

In this subsection, an alternative representation of Caplygin systems will be given and called as the second form. It will be useful in the study of practical systems.

In the remainder of this paper, we always assume that (C1) holds. Thus, the constraints function $J$ can be written as $J\left(z_{1}, z_{2}\right)=\left[\sum_{j=1}^{\bar{n}_{2}} J_{1 j}\left(z_{1}\right) z_{2 j} \quad \bar{J}_{2}\left(z_{1}\right)\right]$. For each $1 \leq j \leq \bar{n}_{2}$, let $\widetilde{J}_{1 j}$ be defined as follows:

$$
\tilde{J}_{1 j}=J_{1 j}-\partial J_{2 j} / \partial z_{1},
$$

where $J_{2 j}$ is $j$-th column vector of $\bar{J}_{2}$. Consider the following coordinate transformation:

$$
\tilde{q}_{2}=q_{2}+\bar{J}_{2}\left(z_{1}\right) z_{2} \text {. }
$$

Then, we have

$$
\begin{aligned}
& \dot{\widetilde{q}}_{2}=\dot{q}_{2}+\bar{J}_{2} \dot{z}_{2}+\sum_{j=1}^{\bar{n}_{2}}\left(\partial J_{2 j} / \partial z_{1}\right) z_{2 j} \dot{z}_{1} \\
& =-\sum_{j=1}^{\bar{n}_{2}} J_{1 j} z_{2 j} \dot{z}_{1}-\bar{J}_{2} \dot{z}_{2}+\bar{J}_{2} \dot{z}_{2}+\sum_{j=1}^{\bar{n}_{2}}\left(\partial J_{2 j} / \partial z_{1}\right) z_{2 j} \dot{z}_{1} \\
& =-\sum_{j=1}^{\bar{n}_{2}} \widetilde{J}_{1 j} z_{2 j} \dot{z}_{1}=-\tilde{J}_{1},
\end{aligned}
$$

where

$$
\widetilde{J}\left(z_{1}, z_{2}\right)=\left[\sum_{j=1}^{\bar{n}_{2}} \widetilde{J}_{1 j}\left(z_{1}\right) z_{2 j} \quad 0\right] .
$$

In new coordinate $\left(q_{1}, \dot{q}_{1}, \widetilde{q}_{2}\right)$, the transformed system is still a Caplygin system in the form (1)-(2). For the convenience, it can be called as the second form of Caplygin systems. Moreover, (C1) also holds with $\bar{J}_{2}=0$ by (35). Then, Theorem 1 can be used to study the exponential stabilizability for new system. We summarize the previous discussions into the following proposition. 
Proposition 3. Consider a Caplygin system of the form (1)(2). Suppose (C1) holds. Using the new coordinate $\left(q_{1}, \dot{q}_{1}, \widetilde{q}_{2}\right)$ with $\widetilde{q}_{2}$ being defined as (34), the transformed system is also a Caplygin system of the form (1)-(2) and (C1) still holds with the new constraint function described as (33) and (35).

In Example 2, it was shown that (C2) does not hold for rolling wheel system under some coordinate transformation. In the following, let us show that $(\mathrm{C} 2)$ becomes true by employing the new transformation.

Example 3. (Rolling wheel systems: revisited). Let us try to verify (C2) for the second form of Caplygin system described in Example 2. According to (32)-(33), the new constrain function $\widetilde{J}=\left[\widetilde{J}_{11} z_{2} \quad 0\right]$ where

$$
\begin{aligned}
& \widetilde{J}_{11}=J_{11}-\partial \bar{J}_{2} / \partial z_{1}=\left[\begin{array}{c}
\sin \left(z_{1}\right) \\
\cos \left(z_{1}\right)
\end{array}\right] \text {. } \\
& \text { In this case, } \bar{J}_{2}=0 \quad \text { and } \\
& \bar{J}_{1}\left(z_{1}, z_{3}\right)=\widetilde{J}_{11}\left(z_{1}\right) z_{3}=\left[\sin \left(z_{1}\right) z_{3}, \cos \left(z_{1}\right) z_{3}\right]^{T}, \quad \forall z_{1} \in \mathfrak{R}, \forall z_{3} \in \mathfrak{R} . \\
& \text { Thus, } \quad \bar{P}_{2}=0 \quad, \quad\left[p_{11}^{\bar{J}_{1}}, p_{21}^{\bar{J}_{1}}\right]^{T}=\left[z_{1} z_{3}, z_{3}\right]^{T} \quad \text { and } \\
& \bar{d}=\left(\bar{d}_{1}, \bar{d}_{2}\right)^{T}=\left(\tilde{d}_{1}^{\bar{J}_{1}}, \tilde{d}_{2}^{\bar{J}_{1}}\right)^{T}=(2,1)^{T} \text {. Then, condition (b) in } \\
& \text { Proposition } 2 \text { holds. Moreover, } \\
& \begin{array}{l}
\bar{P}(\eta)=\bar{P}_{1}(\eta)-\left(D_{\bar{d}}\right) \bar{P}_{2}(\eta)=\bar{P}_{1}(\eta)=\left[\bar{p}_{11}^{\bar{d} 0 \bar{J}_{1}}(\eta, \eta), \bar{p}_{21}^{\bar{d} 0 \bar{J}_{1}}(\eta, \eta)\right]^{T} \\
=\left[p_{11}^{\bar{J}_{1}}(\eta, \eta), p_{21}^{\bar{J}_{1}}(\eta, \eta)\right]^{T}=\left[\eta^{2}, \eta\right]^{T}, \forall \eta \in \mathfrak{R} .
\end{array}
\end{aligned}
$$

Therefore, (a) in Proposition 2 also holds for any $\eta_{0} \neq 0$. Thus, the origin is globally $\rho$-exponentially stabilizable for the new system by Theorem 1 and Proposition 2 .

Remark 1. At glance over (C2), it is like a controllability condition and seems invariant under various coordinate transformations. Unfortunately, it is not true in general as it was seen in the example of rolling wheel system. That is to say that (C2) depends on the used coordinate transformations!! This phenomenon points out a fact that a suitable representation form is important for the exponential stabilization of nonholonomic systems as reflected in present literature.

\section{CONCLUSIONS}

The global $\rho$-exponential stabilizability of the origin for nonholonomic Caplygin systems was guaranteed based on a simplified controllability condition. The proposed criteria are easily checked and simpler than the previous result given in [11]. Furthermore, an interesting coordinate transformation (second form) of Caplygin systems was also given so that the proposed criterion can be applied to various situations. Several illustrated examples were given to validate the effectiveness of our approaches. The future work may toward to deduce a similar result for a more general class of nonholonomic systems [16]. On the other hand, the robustness for the proposed controllers is also interesting and deserves more discussions in view of the recent result given in [7].

\section{ACKNOWLEDGMENT}

This work was supported by the NSC, Taiwan, R.O.C., under contracts NSC 92-2213-E-159-001 and NSC 922212-E-001-001.

\section{REFERENCES}

[1] A. Astolfi, "Discontinuous control of nonholonomic systems," Systems and Control Letters, vol. 27, pp. 37-45, 1996.

[2] A. M. Bloch, N. H. McClamroch and M. Reyhanoglu,"Control and stabilizability of nonholonomic Caplygin dynamic systems," in Proceedings of the IEEE Conf. Decision Control, Brighton, U.K., 1991, pp. 1127-1132.

[3] A. M. Bloch, M. Reyhanoglu and N. H. McClamroch,"Control and stabilizability of nonholonomic dynamic systems ," IEEE Trans. on Automatic Control, vol. 37, pp. 1746-1757, 1992.

[4] W. Brockett, "Asymptotic stability and feedback stabilization," in: R. W. Brockett, R. S. Millman, \& H. J. Sussmann Eds, Differential geometric control theory, 1983, pp. 181-191.

[5] C. T. Chen, Introduction to linear system theory, New York: Holt, Rinehart and Winston, 1986.

[6] J. M. Godhavn and O. Egeland, "A Lyapunov approach to exponential stabilization of nonholonomic systems in power form," IEEE Trans. on Automatic Control, vol. 42, pp. 1028-1032, 1997.

[7] Z. P. Jiang, "Robust exponential regulation of nonholonomic systems with uncertainties," Automatica, vol. 36, pp. 189-209, 2000.

[8] I. Kolmanovsky and N. H. McClamroch, "Developments in nonholonomic control problems," IEEE Control Systems Magazine, vol. 15, pp. 20-36, 1995.

[9] I. Kolmanovsky and N. H. McClamroch, "Stabilization of nonholonomic Chaplygin systems with linear base space dynamics," in Proceedings of the American Control Conference, New Oreleans, LA, 1995, pp. 27-32.

[10]M. C. Laiou and A. Astolfi, "Quasi-smooth control of chained systems," in Proceedings of IEEE Conf. Decision Control, San Diego, California, 1999, pp. 3940-3944.

[11]T. C. Lee, "Exponential stabilization for nonlinear systems with applications to nonholonomic systems," Automatica, vol. 39, pp. 1045$1051,2003$.

[12]R. T. M'Closkey and R. M. Murray, "Exponential stabilization of driftless control systems using homogeneous feedback," IEEE Trans. on Automatic Control, vol. 42, pp. 614-628, 1997.

[13]R. M. Murray and S. Sastry, "Nonholonomic motion planning: steering using sinusoids," IEEE Trans. on Automatic Control, vol. 38, pp. 700$716,1993$.

[14]M. Reyhanoglu, "Exponential stabilization of an underactuated autonomous surface vessel," Automatica, vol. 33, pp. 2249-2254, 1997.

[15]M. Reyhanoglu, S. Cho, N. H. McClamroch and I. Kolmanovsky, "Discontinuous feedback control of a planar rigid body with an unactuated degree of freedom," in Proceedings of IEEE Conf. Decision Control, Tampa, Florida, 1998, pp. 433-438.

[16]M. Reyhanoglu, A. J. van der Schaft, N. H. McClamroch and I. Kolmanovsky, "Dynamics and control of a class of underactuated mechanical systems," IEEE Trans. on Automatic Control, vol. 44, pp. 1663-1671, 1999

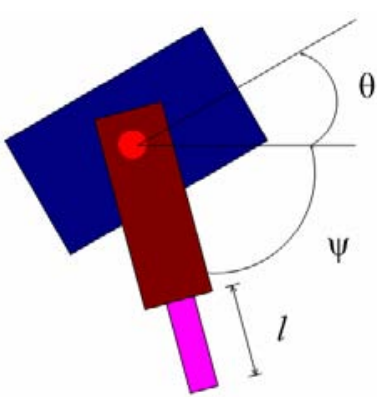

Fig. 1: An example of Caplygin system: hopping robot. 